Service social

\title{
Le discours des défenseurs des droits des hommes sur la violence conjugale \\ Une analyse critique
}

\section{Normand Brodeur}

Volume 50, numéro 1, 2003

URI : https://id.erudit.org/iderudit/006925ar

DOI : https://doi.org/10.7202/006925ar

Aller au sommaire du numéro

\section{Éditeur(s)}

École de service social de l'Université Laval

ISSN

1708-1734 (numérique)

Découvrir la revue

Citer cet article

Brodeur, N. (2003). Le discours des défenseurs des droits des hommes sur la violence conjugale : une analyse critique. Service social, 50(1), 145-173. https://doi.org/10.7202/006925ar
Résumé de l'article

Se disant victimes d'injustices non reconnues, les défenseurs des droits des hommes dénoncent le discours féministe sur la violence masculine et les politiques sociales mises en place depuis vingt ans pour combattre la violence conjugale. Cet article résume leurs principaux arguments à partir d'une analyse de contenu de trois ouvrages publiés au Québec. La seconde partie de l'article situe ces arguments à l'intérieur des controverses autour de la définition de la violence, de la violence des femmes et du maintien d'un équilibre entre les droits des victimes et ceux des personnes accusées devant les tribunaux. Elle fait aussi état de données qui remettent en question les thèses des défenseurs des droits des hommes sur la symétrie générale de la violence entre les sexes et sur la discrimination systématique dont les hommes seraient victimes au sein du système judiciaire. 


\title{
Le discours des défenseurs des droits des hommes sur la violence conjugale Une analyse critique
}

\author{
Normand BRODEUR \\ Étudiant au doctorat \\ École de service social \\ Université Laval \\ normand.brodeur@sympatico.ca
}

Se disant victimes d'injustices non reconnues, les défenseurs des droits des hommes dénoncent le discours féministe sur la violence masculine et les politiques sociales mises en place depuis vingt ans pour combattre la violence conjugale. Cet article résume leurs principaux arguments à partir d'une analyse de contenu de trois ouvrages publiés au Québec. La seconde partie de l'article situe ces arguments à l'intérieur des controverses autour de la définition de la violence, de la violence des femmes et du maintien d'un équilibre entre les droits des victimes et ceux des personnes accusées devant les tribunaux. Elle fait aussi état de données qui remettent en question les thèses des défenseurs des droits des hommes sur la symétrie générale de la violence entre les sexes et sur la discrimination systématique dont les hommes seraient victimes au sein du système judiciaire.

Mots clés : Québec, droits des hommes, violence conjugale, analyse de contenu

Claiming that they are the victims of unacknowledged injustices, men's rights activists denounce both the feminist discourse on men's violence and the social policies that were implemented in the last 20 years to end domestic violence. This article presents a synthesis of their main arguments based on a content analysis of three books published in Quebec. The second part of the article places these arguments within existing controversies about the definition of violence, violence by women, and the balance between the rights of the victims and the rights of those who are accused of domestic violence. It also reports data that cast doubt on the men's rights activists claims that violence between men and women is generally symmetrical and 
that men are victims of systematic discrimination within the criminal justice system.

Key words: Québec, men's rights, domestic violence, content analysis 
Dans les années 1990, plusieurs auteurs ont proposé une analyse des principaux discours sur la masculinité (Clatterbaugh, 1997 et 2000; Dulac, 1991; Messner, 1998). Ces travaux montrent que les hommes québécois et nord-américains n'ont pas réagi de façon uniforme à l'émergence du mouvement féministe au cours des trente dernières années. II n'y a pas, en effet, de mouvement des hommes homogène. Certains ont épousé la cause des femmes avec enthousiasme et ont revendiqué le nom de " proféministes ", voire de féministes. D'autres se sont inspirés des premières analyses féministes sur les contraintes liées aux rôles sexuels stéréotypés pour réfléchir à leur propre situation. Tout en reconnaissant l'oppression des femmes, ils ont mis en relief les contraintes sociales associées à la masculinité traditionnelle et ont tenté de s'en libérer.

Les analystes signalent aussi la présence d'un troisième type de discours centré sur la défense des droits des hommes. Les auteurs et les groupes qui s'inscrivent dans ce courant de pensée affirment qu'ils font l'objet d'injustices non reconnues, tant sur le plan légal, social que psychologique, et qu'ils constituent les nouvelles victimes du sexisme dans la société contemporaine. Par conséquent, ils s'organisent afin de défendre les droits des hommes dans les causes de divorce, mettent en doute la pertinence des programmes de discrimination positive en faveur des femmes et dénoncent les publicités qui véhiculent une image négative de l'homme.

Les défenseurs des droits des hommes critiquent également un certain nombre de thèses au sujet de la violence masculine en général et de la violence conjugale en particulier, ces questions ayant à leur avis été galvaudées par le mouvement féministe (Côté, 1990). Cette partie de leur discours constitue une vigoureuse dénonciation des pratiques et des politiques sociales mises en place pour combattre la violence conjugale et doit, à ce titre, être connue des personnes qui interviennent dans ce champ. C'est pour contribuer à cette connaissance que le présent article a été rédigé. II présente d'abord une analyse thématique permettant de saisir la logique interne de l'argumentation. II propose ensuite quelques réflexions critiques sur trois dimensions clés du discours, soit la façon de définir et d'expliquer la violence conjugale, la symétrie présumée dans la violence des hommes et des femmes ainsi que la réponse du système judiciaire. 


\section{Méthodologie}

À l'origine, l'analyse présentée ici constituait un exercice universitaire réalisé dans le cadre d'un cours sur la condition masculine. Elle a par la suite été développée davantage en vue d'une série de présentations publiques ${ }^{1}$. Compte tenu du temps et des ressources restreintes dont nous disposions, nous avons limité l'étude du discours à des monographies dans lesquelles les auteurs expriment leur point de vue avec plus de détails que dans des articles de journaux ou de revues. Trois ouvrages ont ainsi été retenus. Le premier est le Manifeste d'un salaud de Roch Côté (1990). Ce livre au style pamphlétaire $^{2}$ a été écrit à la suite des événements dramatiques survenus à l'École Polytechnique de Montréal le 6 décembre 1989. L'auteur y déconstruit, dénonce et tourne en dérision les arguments d'auteures féministes qui ont vu dans l'assassinat des 14 jeunes femmes l'expression extrême d'une violence exercée quotidiennement contre les femmes. Le second ouvrage, intitulé Coupable d'être un homme: violence conjugale et délire institutionnel, a été écrit par Georges Dupuy (2000), un militant du Groupe d'entraide aux pères et de soutien à l'enfant (GEPSE). L'auteur s'élève contre la Politique d'intervention gouvernementale en matière de violence conjugale (Gouvernement du Québec, 1995), ainsi que contre les pratiques du système pénal envers les hommes accusés dans une affaire de violence conjugale. Le dernier ouvrage est Homme et fier de l'être, d'Yvon Dallaire (2001). Ce livre dénonce les préjugés contre les hommes et s'intéresse à l'épanouissement de l'homme dans le couple et dans la société. Bien que la question de la violence ne constitue pas l'objet principal du livre, elle y occupe une place importante ${ }^{3}$. À notre connaissance, ces livres sont les seuls ouvrages québécois destinés au grand public à avoir abordé in extenso la question de la violence

1. Notamment au colloque "Peut-on légitimer ou excuser l'usage de la violence ?» tenu en novembre 2002 et organisé conjointement par l'organisme Après-Coup, le Centre jeunesse de Montréal et les Centres jeunesse de la Montérégie.

2. Le Petit Robert (1989) définit un pamphlet comme étant un court écrit satirique, qui attaque avec violence le gouvernement, les institutions, la religion, un personnage connu.

3. Signe de l'importance du thème, l'auteur a publié en 2002 un condensé des thèses sur la violence conjugale qu'il avait déjà présentées dans son ouvrage de 2001. Voir Yvon Dallaire, La violence faite aux hommes. Une réalité taboue et complexe, Québec, Les Éditions Option Santé, 2002, 63 p. 
dans une perspective de défense des hommes, ce qui leur confère une importance particulière. II faut toutefois souligner que les défenseurs des droits des hommes expriment aussi leur point de vue dans des articles de journaux, des revues et des sites Internet. L'exclusion de ces sources de données du corpus analysé constitue une limite méthodologique de la présente étude.

L'analyse thématique qui suit a été faite à partir de la grille de lecture dont l'auteur américain Kenneth Clatterbaugh (1997) s'est servi dans son livre Contemporary Perspectives on Masculinity pour définir une typologie des différents points de vue sur la condition masculine. Cette grille comprend les quatre questions suivantes: (a) quelle est, du point de vue des auteurs étudiés, la réalité sociale des hommes? (b) quels sont, selon eux, les facteurs d'explication et de maintien de cette réalité sociale? (c) quelle est la réalité qu'ils jugent souhaitable pour les hommes? et (d) quelles stratégies proposent-ils pour atteindre la réalité souhaitée? Pour appliquer cette grille de lecture aux trois ouvrages retenus, le contenu de ceux-ci a d'abord été numérisé intégralement. Ensuite, le logiciel Nud-Ist (Qualitative Solution and Research, 1997) a été utilisé pour découper les textes en unités d'une ligne. Finalement, chaque unité de texte a été lue et classée dans l'une des quatre catégories analytiques en tenant compte de son contexte.

\section{ANALYSE THÉMATIQUE}

\section{La réalité sociale des hommes}

S'appuyant sur des citations tirées de la littérature féministe, les auteurs étudiés soutiennent que le féminisme a instauré un véritable procès de l'homme que l'on accuse d'être généralement violent et misogyne. Cette accusation, dit Côté (1990), se retrouve chez les auteures qui ont vu dans la démence meurtrière de Marc Lépine une reproduction du comportement normal et quotidien de tous les hommes, ainsi que dans les campagnes de prévention de la violence conjugale où on laisse croire que nous vivons dans un pays de batteurs de femmes. Elle vise tous les hommes, car ces derniers sont perçus comme étant solidaires et complices de la violence, même lorsqu'ils ne sont pas auteurs d'agressions directes contre les femmes. Dallaire (2001) abonde dans le même sens en affirmant que tous les hommes sont victimes de préjugés qui les présentent comme des êtres violents, des violeurs en puissance ou des abuseurs d'enfants. 
Selon les auteurs, l'homme est aussi accusé d'être dévoré par la soif du pouvoir, de ne pas avoir de respect pour la femme et de dégrader la nature constamment. On lui attribue de plus l'entière responsabilité des guerres, des massacres, du totalitarisme et de tous les autres maux qui affligent l'humanité. Selon ce que Côté (1990, p. 36) appelle "le plaidoyer féministe ", cet état de choses tiendrait aux valeurs des hommes qui privilégient l'agression, la domination et la destruction à toute autre chose. L'antidote à ce mal universel serait d'accroître la présence des femmes dans toutes les sphères de l'activité humaine :

À entendre [les femmes], [...] l'homme serait le côté sombre de l'humanité, alors que la femme en serait le côté lumineux, celle qui sait mieux que lui comment les choses devraient fonctionner. II faudrait donc que la femme prenne non seulement le contrôle de la famille, de l'éducation des enfants, de la gestion des soins, mais qu'elle prenne aussi le contrôle économique et politique de la société pour qu'advienne une véritable civilisation humaine. (Dallaire, 2001, p. 17.)

Pour les auteurs, ce procès symbolique ternit la réputation de l'homme et a plusieurs effets néfastes sur lui. Ainsi, Dallaire (2001) mentionne que les pères sont perçus comme des menaces pour leurs enfants, que les garçons n'ont plus de modèles positifs auxquels s'identifier, que les filles se méfient des garçons et que les femmes perçoivent la sexualité masculine comme agressive et violente.

Les auteurs situent le procès intenté à l'homme dans le contexte d'une guerre des sexes qui fait rage autour d'enjeux comme le partage des tâches domestiques, la décision d'avoir ou non des enfants et la négociation des besoins de chacun. Bien que cette guerre se déroule dans le quotidien des couples, c'est dans les situations de rupture et de divorce qu'elle atteint son paroxysme. La garde des enfants, le partage des biens et la perte du sens à la vie deviennent alors des enjeux « si importants qu'ils font oublier toute prudence aux belligérants » (Dupuy, 2000, p. 73). Pour les auteurs étudiés, il ne fait pas de doute que les hommes sont les grands perdants de la guerre entre les sexes. Ces auteurs estiment que le divorce, motivé de plus en plus par des raisons égoïstes (voir, par exemple, Dallaire, 2001, p. 141), a un impact psychologique plus lourd pour les hommes que pour les femmes qui, dans la majorité des cas, en sont les instigatrices. Cette situation déjà difficile serait aggravée par les injustices 
que les hommes subissent au moment des divorces. À ce propos, Dupuy (2000) affirme que les femmes peuvent maintenant obtenir facilement la garde des enfants et s'approprier les biens du couple en faisant de fausses allégations de violence conjugale. II n'est pas clair dans les propos de l'auteur si cette tactique est le fait de quelques femmes "qui exploitent à leur avantage un système vicié » (p. 11) ou si le " coup monté, préparé et soigneusement orchestré » est "typique " des situations où des accusations de violence conjugale sont portées (p. 18). Les verdicts de culpabilité non fondés seraient toutefois en forte augmentation (p. 84).

Pour Dupuy (2000), les femmes qui font de fausses allégations de violence tentent d'utiliser les moyens mis à leur disposition pour améliorer leur position stratégique. Ce qui est plus grave à ses yeux, c'est que le système de justice défend inconditionnellement leur point de vue au lieu de faire éclater la vérité. Les hommes, dit-il, sont "broyés » (p. 35) par ce système qui présume de leur culpabilité avant même qu'ils aient ouvert la bouche (p. 70), qui s'appuie sur un arsenal de moyens pour les éloigner de leur famille et qui les prive d'une défense pleine et entière. En plus de dénoncer l'introduction dans le Code criminel du délit de harcèlement, le traitement des dossiers par procédure sommaire, l'utilisation fréquente de l'article 810 du Code criminel $^{4}$ et les restrictions dans l'accès à l'aide juridique, l'auteur critique le parti pris des acteurs du système pénal. II reproche aux policiers de bâcler les enquêtes, aux juges d'errer dans l'interprétation des faits, aux substituts du Procureur de la couronne de terroriser les hommes en les menaçant de procédures supplémentaires et aux avocats de la défense de plaider sans conviction. Les injustices dont les hommes sont victimes de la part du système judiciaire sont à son avis le fruit d'un "véritable complot » ( $p .35$ ) qu'il assimile à la persécution des juifs par les nazis (p. 10), au maccarthysme (p. 175), à la chasse aux sorcières (p. 56) et au « goulag judiciaire » (p. 71).

4. L'article 810 permet à une personne de déposer une dénonciation devant un juge de paix si elle craint, pour des motifs raisonnables, qu'une autre personne ne lui cause des lésions personnelles ou n'endommage sa propriété. Le juge de paix peut alors ordonner que le défendeur prenne l'engagement de ne pas troubler l'ordre public, d'observer une bonne conduite et de respecter certaines conditions pendant une période maximale de 12 mois (Code criminel du Canada, L. R. 1985, ch. 46). 


\section{L'explication de la réalité}

Selon les auteurs étudiés, plusieurs facteurs expliquent que les hommes soient soumis à un procès symbolique et à des accusations criminelles injustes. Tous s'entendent toutefois pour en attribuer la responsabilité première à un féminisme qu'ils qualifient de « doctrinaire ", " extrémiste ", " intégriste » ou " exacerbé ». Le courant radical du mouvement des femmes a, disent-ils, trahi les objectifs initiaux du féminisme et en a érigé la doctrine en système fermé. Les trois auteurs se défendent cependant d'être antiféministes. Ils reconnaissent l'existence d'injustices historiques à l'égard des femmes et valorisent le fait qu'elles se soient organisées pour y mettre un terme. Pour Côté (1990), leur lutte pour l'acquisition et l'exercice de droits tels que l'équité salariale s'inscrit dans la logique de la démocratie libérale et ne peut être contestée. Par ailleurs, un féminisme " doux » peut contribuer au développement d'une société plus égalitaire où les individus seront plus autonomes, les couples plus intimes et les enfants plus heureux, l'homme et la femme étant tous les deux gagnants (Dallaire, 2001, p. 307). Le problème ne tient donc pas aux objectifs égalitaristes du féminisme, mais bien au fait que celui-ci soit allé trop loin en visant la prédominance des femmes dans la société (Dupuy, 2000, p. 106). Le féminisme " extrémiste » serait ainsi devenu une nouvelle dictature cherchant à corriger une injustice en en créant une autre et à " faire payer aux méchants tout ce qu'ils ont fait aux victimes » (Dallaire, 2001, p. 19).

Selon Côté (1990), le féminisme " doctrinaire » repose sur un mode de pensée manichéen qui divise le monde entre bons et méchants, l'homme faisant office de "Grand Satan » (p. 36). Cet argument central est repris par les deux autres auteurs qui rejettent toute vision du monde qui dépeint systématiquement les hommes comme des agresseurs et les femmes comme des victimes (Dallaire, 2001, p. 87) en ignorant les situations dites intermédiaires (Dupuy, 2000, p. 124). Tous soulignent que la pensée dichotomique déforme les faits en passant sous silence la contribution positive des hommes à la famille et à la société. Côté poursuit par ailleurs sa critique du féminisme " doctrinaire » en lui reprochant de s'imposer par la culpabilisation. En invoquant la faute collective, dit-il, les féministes cantonnent tous les hommes dans un rôle de salaud. Ceux-ci sont ensuite condamnés au silence, puisque toute personne qui remet la "parole sacrée » en question risque selon l'auteur d'être vilipendée sur la place publique et accusée de misogynie (p. 18). 
Le féminisme " extrémiste » a pu s'imposer, disent les auteurs, parce qu'il a bénéficié de la complicité d'hommes proféministes. Dallaire (2001) présente ces derniers comme des hommes qui, ne pouvant " sentir leurs semblables » (p. 67), se mettent volontairement du côté des féministes pour combattre leur propre sexe; ce sont, en d'autres termes, "des loups dans la bergerie » (p. 71). Côté (1990) propose le terme de "compagnons de route " et résume ainsi le rôle de ces derniers dans le procès intenté contre les hommes:

Le compagnon de route est une conscience malheureuse. II est né du mauvais bord, celui des salauds. Et sa vie consistera à racheter cette faute originelle. [...] La culpabilité existentielle du compagnon de route en fait un imprécateur redoutable, un être retentissant de repentir et d'anathème. II est en général plus méchant que ceux qu'il accompagne. Les chiens et les salauds n'ont qu'à bien se tenir! (Côté, 1990, p. 21.)

Le féminisme " extrémiste " bénéficie aussi de la complicité de politiciens et d'institutions sociales que les auteurs dénoncent au passage. Ces derniers critiquent notamment: (a) les programmes d'aide aux conjoints aux comportements violents qui, selon Dupuy (2000, p. 102), visent à faire croire à ces derniers qu'ils sont coupables et à justifier la répression à leur égard; (b) les politiciens qui accordent des budgets dérisoires aux groupes venant en aide aux hommes en difficulté (Dallaire, 2001, p. 72); et (c) les médias qui font la promotion des valeurs féminines (Dallaire, p. 154) et reprennent sans discernement le discours féministe sur la violence conjugale (Côté, 1990, p. 94).

Bien qu'ils considèrent l'emprise de l'idéologie féministe comme le premier facteur d'explication de la réalité qu'ils décrivent, les auteurs fondent aussi leur critique sur d'autres arguments. Ainsi, pour Côté (1990), le discours sur la violence masculine généralisée témoigne d'une obsession grandissante pour la sécurité dans les sociétés occidentales. L'individu moderne, dit-il, amplifie tous les risques et c'est dans les milieux " douillets » de la bourgeoisie syndicale et universitaire, où « le narcissisme est cultivé comme un art de vivre » (p. 70), que l'on trouve les plus fortes réactions à la violence. Plus la société s'adoucit, plus les gestes de violence prennent du relief et deviennent intolérables aux yeux des gens, le résultat étant que les sociétés où la violence est la plus faible sont celles où on la dénonce le plus. Dans un autre ordre d'idées, Dupuy (2000, p. 72-74) soutient 
que le système judiciaire accusatoire nord-américain est vulnérable aux fausses accusations. Les plaignantes de mauvaise foi y ont la partie facile, dit-il, puisqu'elles n'ont qu'à alléguer une agression sexuelle, de la violence conjugale ou du harcèlement criminel pour que se mettent en branle des procédures judiciaires contre leur conjoint. Et elles n'ont pas à subir de conséquences en cas d'accusations mensongères.

Les auteurs expliquent finalement le maintien des injustices faites aux hommes par leur propre faiblesse. Habités par des sentiments de honte, de culpabilité et de peur, ils ne réagissent pas avec la vigueur qu'il faudrait aux accusations dont ils sont victimes. L'homme moyen, envahi par la honte d'être du côté des exploiteurs, cherche la rédemption dans la passivité et le silence face au discours féministe (Côté, 1990, p. 21). L'homme qui doit faire face à des procédures judiciaires injustes se laisse pour sa part manipuler et fait tout pour tenter d'étouffer l'affaire dans laquelle il est impliqué (Dupuy, 2000, p. 114).

\section{La réalité sociale souhaitable}

Les auteurs étudiés veulent restaurer l'image de l'homme ternie par le féminisme "doctrinaire » et mettre fin au sexisme dont les hommes sont victimes. Pour atteindre ces buts, ils cherchent à redéfinir les réalités décrites par les féministes et proposent leur propre projet de société.

\section{La redéfinition de la réalité}

Les auteurs remettent en question une notion clé du féminisme, celle du patriarcat. Pour Côté (1990), il n'y a dans la société québécoise aucune misogynie triomphante, les hommes d'ici ayant plutôt la réputation d'être parmi "les moins machos de la terre » (p. 25). Bien qu'une minorité d'entre eux se sentent menacés par l'avancée des femmes, la majorité a accepté qu'elles jouent un rôle équivalent au leur dans la société. Par conséquent, dit l'auteur, croire que les hommes souhaitent remettre les femmes à leur place et que Marc Lépine a tiré en leur nom à l'École Polytechnique relève de la fabulation. Par ailleurs, les auteurs analysés ne croient pas que les hommes constituent un groupe privilégié par rapport aux femmes, dont l'espérance de vie est en moyenne de six ou sept ans supérieure à celle des hommes (Côté, 1990; Dallaire, 2001). Ils ajoutent que la vie des hommes a été sacrifiée au profit des femmes et des enfants tout 
au long de l'Histoire, soulignant qu'il n'y a pas de privilège évident associé au fait de risquer sa vie à la guerre, d'exercer des métiers périlleux ou d'user sa santé au travail pour assurer la subsistance de la famille. Dallaire (2001, p. 58) va encore plus loin dans sa critique de la notion de patriarcat en soutenant que "les hommes ont peutêtre le pouvoir social, politique, juridique et économique, mais que le vrai pouvoir, le pouvoir occulte, est entre les mains des femmes ».

Les trois auteurs reconnaissent que le problème des femmes victimes de violence conjugale est réel. Ils estiment cependant qu'il a été mal posé par le mouvement féministe et s'emploient à le redéfinir. Ils critiquent d'abord la définition de la violence conjugale qui est à leur avis beaucoup trop large :

La notion de violence a évolué dans nos sociétés. Alors qu'elle fut longtemps associée à la brutalité, à la force physique et à des comportements extrêmes, elle est maintenant, selon certains groupes, de plus en plus définie à partir d'une simple relation de domination ou de tentative de contrôle. [...] Violence "économique », violence "psychologique », violence "verbale ", l'aire sémantique du mot "violence » a tellement été étirée qu'on peut y mettre à peu près n'importe quel comportement. (Dupuy, 2000, p. 122-123.)

Pour Côté (1990), il ne saurait être question d'étendre ainsi le sens du mot "violence », dont la définition doit être limitée au meurtre, au viol et aux coups et blessures (p. 71).

Les féministes considèrent la violence comme un instrument de domination et dénoncent la tendance des hommes à vouloir contrôler leur partenaire. Dallaire (2001, p. 211) s'oppose à cette analyse de deux façons. II considère d'abord que les hommes entrent naturellement en relation selon un mode hiérarchique. S'ils veulent ordonner les choses, dit-il, c'est pour qu'elles fonctionnent bien et non pas pour dominer leur partenaire. L'auteur attribue ensuite à la violence une fonction expressive. L'homme, dit-il, envoie des messages à sa partenaire avant de passer aux gestes violents. Ce n'est que lorsque ces signaux ne sont pas entendus qu'il "s'affirme " par la violence physique (p. 97). "Bardasser », fermer les portes et panneaux d'armoire avec force, voire frapper sa partenaire constituent des moyens par lesquels il exprime sa frustration (p. 109). 
Par ailleurs, les trois auteurs contestent les statistiques selon lesquelles les femmes sont les principales victimes de la violence au sein du couple. Les études féministes sont biaisées, disent-ils, parce que leurs échantillons ne sont pas représentatifs, que l'on ne pose pas les mêmes questions aux hommes et aux femmes et que la définition très large de la violence gonfle le phénomène. Au-delà de ces considérations méthodologiques, les auteurs mettent en doute la bonne foi des chercheures féministes à qui ils reprochent de tripoter les statistiques, de les fabriquer de toutes pièces afin d'arriver à des conclusions déjà tirées d'avance (Côté, p. 24). Les études dont les résultats ne sont pas "politiquement corrects", comme celles qui mettent en lumière la tendance des femmes à la violence, sont à leur avis rejetées et cachées au public (Dallaire, 2001, p. 126; Dupuy, 2000, p. 94).

Après avoir mis en doute la valeur des statistiques produites par la recherche féministe, les auteurs concluent que la violence des hommes est sur-évaluée, alors que celle des femmes est sous-évaluée. En réalité, disent-ils, la violence des hommes et des femmes est parfaitement symétrique, tant en fréquence qu'en sévérité. Cette thèse sur la symétrie de la violence est illustrée dans la façon dont les auteurs traitent la question de l'homicide conjugal. S'appuyant sur des statistiques américaines des années 1970, Dallaire (2001, p. 124) soutient qu'il y a autant d'hommes que de femmes tués par leur conjoint. Dupuy (2000, p. 107) cite des statistiques canadiennes plus récentes indiquant que les femmes sont victimes des trois quarts des homicides conjugaux. II estime cependant qu'il faut corriger ces statistiques en y ajoutant les meurtres que les femmes ont commandités en faisant appel à un intermédiaire pour tuer leur conjoint (p. 135). II propose aussi de considérer simultanément les homicides et les suicides. En calculant ainsi le nombre de personnes qui décèdent des suites de la violence conjugale, dit-il, on en arriverait à reconsidérer le déséquilibre apparent, puisque les hommes se suicident beaucoup plus souvent que les femmes et que plusieurs d'entre eux le font " après avoir été privés sans raison valable de leurs enfants et de leur maison, ou après avoir été emprisonnés sans motif » (p. 108).

\section{Le projet social}

La société à laquelle les auteurs aspirent devra reconnaître davantage « ce que les hommes ont fait, font et continueront de faire pour 
l'amélioration de l'Humanité »(Dallaire, 2001, p. 34). Pour cela, il faudra : (a) que le féminisme s'adoucisse en reconnaissant la coresponsabilité des femmes dans l'histoire de l'humanité et en cessant de diviser le monde en bourreaux et victimes; (b) que les hommes s'affirment et refusent de se définir en fonction des attentes des femmes; et (c) que l'on renouvelle le discours sur les hommes en s'intéressant à eux « autrement qu'à travers les clichés véhiculés par un féminisme sommaire et vindicatif » (Côté, 1990, p. 219).

Sans faire l'apologie de la violence, Dupuy (2000, p. 123) plaide pour une société plus tolérante aux agressions physiques mineures et aux formes de violence non physique qui lui semblent inévitables dans un contexte de guerre des couples, où les rapports de domination sont inhérents à la vie conjugale. Concrètement, il demande que les condamnations soient moins systématiques dans les cas de harcèlement ou de menaces de mort où il y a place à l'interprétation des mots. II souhaite également que les juges soient plus critiques lorsque les accusations de voies de fait concernent des «peccadilles » telles que pousser, repousser ou retenir une personne (p. 112).

Considérant les fausses allégations de violence conjugale et le parti pris des institutions en faveur des femmes comme des menaces pour la démocratie, Dupuy (2000) veut éviter les dérapages et protéger les droits des personnes accusées. II revendique donc le retrait complet de la politique gouvernementale d'intervention en matière de violence conjugale, ainsi que de toute politique similaire fondée sur le principe de la tolérance zéro. II réclame également une série de modifications au fonctionnement de l'appareil de justice, par exemple: le retrait des directives données aux policiers pour l'arrestation systématique des hommes lorsqu'il y a allégations de violence conjugale; l'exigence de preuves directes pour justifier les arrestations; la prise en note par les policiers des démentis de l'accusé; la prise en compte de la notion de doute raisonnable par les juges; la justification des décisions du tribunal par écrit; la tenue systématique des enquêtes préliminaires auxquelles les accusés ont droit; la possibilité pour les accusés de contre-interroger eux-mêmes la plaignante; et le retrait de l'article 810 du Code criminel.

\section{Le programme d'action}

Le programme d'action proposé par les auteurs se compose de stratégies individuelles et collectives. Ainsi, pour contrer la culpabilisation collective dont les hommes sont victimes, Côté (1990) et Dallaire 
(2001) en appellent à l'éthique individuelle. Les hommes doivent refuser le procès qui leur est fait, cesser de se sentir coupables des violences exercées par les autres et s'affirmer davantage face à leurs partenaires. Ils doivent toutefois se montrer responsables de leurs pensées, de leurs attitudes, de leurs paroles, de leurs gestes et des conséquences qui en découlent. Ils doivent respecter les différences, s'engager et renoncer à une partie d'eux-mêmes au profit du couple. Par ailleurs, les hommes faussement accusés de violence conjugale devront, selon Dupuy (2000), défendre leur cause avec détermination, en assurant si possible leur propre défense et en utilisant tous les recours dont ils disposent. Cela inclut notamment des poursuites civiles contre la plaignante, les corps policiers et le Procureur général lorsque les accusations s'avèrent sans fondements.

Sur un plan collectif, les auteurs proposent quatre stratégies d'action complémentaires. Premièrement, les hommes doivent dénoncer publiquement le discours féministe sur la violence masculine et la violence conjugale en publiant des livres consacrés à la cause des droits des hommes ou en utilisant d'autres médias. Dupuy (2000, p. 137-138) estime notamment que la presse écrite constitue un véhicule privilégié pour livrer ce type de message. Le deuxième élément de la stratégie consiste à mettre sur pied des associations vouées à la défense des droits des hommes dont la mission consiste, d'une part, à mener la lutte politique et, d'autre part, à donner aux membres des services concrets. Le troisième élément de la stratégie consiste à attirer l'attention des autorités sur la situation des hommes. Dans le cadre de cette lutte politique, divers groupes de défenseurs des droits des hommes ont sollicité la tenue de commissions parlementaires et organisé des manifestations publiques pour dénoncer les politiques gouvernementales d'intervention en matière de violence conjugale. La politique québécoise a même été dénoncée auprès de I'Organisation des Nations Unies (ONU) (Dupuy, 2000). Enfin, le dernier élément de la stratégie proposée se situe sur le terrain de la recherche. Alléguant que les féministes et leurs alliés bénéficient de millions de dollars de fonds publics pour conduire des études biaisées en faveur des femmes, Dupuy (2000) réclame sa part de subventions gouvernementales afin de réaliser ses propres études sur les injustices dont les hommes sont victimes. 


\section{ANALYSE CRITIQUE}

Les auteurs cités dans la première partie de cet article expriment une colère profonde face à l'image que le féminisme radical a dépeinte des hommes et aux politiques sociales mises en place pour contrer la violence faite aux femmes en milieu conjugal. Leurs analyses constituent souvent l'antithèse des thèses féministes. Ils y remplacent le patriarcat par le matriarcat, le sexisme à l'égard des femmes par le sexisme à l'égard des hommes, la violence masculine unidirectionnelle par la violence parfaitement symétrique, la responsabilité collective par la responsabilité individuelle et l'instrumentalité par l'expressivité. Malgré le soutien qu'ils professent à l'endroit des objectifs égalitaires d'un féminisme "doux » et leurs propres mises en garde contre un " hominisme réactionnaire » (Dallaire, 2001, p. 307), leurs propos inquiètent ceux et celles qui soutiennent la cause des femmes victimes de violence. Plusieurs commentateurs qualifient simplement ce type de discours d'antiféministe (Messner, 1998; Strong-Boag, 1996).

En se campant dans l'antithèse féministe de façon rigide, l'idéologie de la défense des droits des hommes peut devenir un filtre déformant aussi puissant que le féminisme "doctrinaire " qu'elle s'emploie à dénoncer. Cependant, au-delà de cette rigidité, le discours des défenseurs des droits des hommes soulève des questions qui ne peuvent être éludées. Cette seconde partie de l'article tente de rendre compte de cette complexité en proposant une critique du discours des auteurs étudiés et en resituant quelques-unes de leurs thèses à l'intérieur des débats qui ont cours dans les milieux de recherche et de pratique intéressés par la problématique de la violence conjugale.

\section{Définition, fonction et explication de la violence}

La définition de la violence conjugale est débattue depuis longtemps par les spécialistes de ce champ d'étude (Rondeau, 1994). Gelles et Straus (1988), des pionniers de l'étude de la violence dans la famille, ont toujours soutenu qu'il fallait inclure dans la définition du mot violence non seulement les gestes les plus graves, mais aussi les formes bénignes d'agression physique. Ces dernières doivent selon eux être considérées comme violentes et mauvaises, parce qu'elles constituent souvent le prélude à une escalade vers des gestes plus graves. On constate donc que les féministes n'ont pas été les seules à étendre la définition du mot violence. Leur contribution spécifique a plutôt été d'ajouter à la définition un ensemble d'autres tactiques 
coercitives permettant à une personne d'exercer le contrôle sur une autre. C'est ainsi que l'abus émotionnel, les menaces, l'intimidation, l'isolement et les abus économiques (Shepard, 1991) ont été considérés comme des éléments constitutifs d'une dynamique de violence.

Une définition étendue de la violence comporte des écueils. Comme le suggèrent Côté (1990) et Dupuy (2000), elle peut inclure des comportements qui paraissent anodins lorsqu'on les considère séparément. De plus, lorsque l'on sort de l'univers des agressions physiques, la notion de violence se confond souvent avec celle d'abus qui demeure difficile à définir et fait appel à une plus grande part de subjectivité. Malgré ces difficultés, la définition étendue de la violence présente des avantages sur le plan de l'analyse des situations vécues au sein du couple. Elle permet notamment de lier ensemble des gestes de nature et d'intensité différentes qui ont néanmoins des effets similaires et cumulatifs sur la personne. À cet égard, les personnes qui travaillent auprès des femmes victimes de violence soutiennent depuis longtemps que la répétition de gestes de violence sape l'intégrité physique et psychologique des victimes autant, sinon plus, que leur simple commission (MacLeod, 1987). C'est en considérant l'ensemble des coups, des menaces et des propos dégradants qu'elles subissent que l'on comprend pourquoi certaines femmes sont terrorisées par leur conjoint. Une analyse de la violence limitée aux coups et blessures évacue cette dimension cruciale et ne permet pas de saisir la dynamique dans laquelle elle se produit.

Comme on l'a vu dans la première partie de cet article, certains défenseurs des droits des hommes conçoivent la violence comme un moyen d'expression. En analysant les situations de violence à partir de ce paradigme, une attention particulière est accordée à la personne qui émet le comportement afin de faire ressortir ses émotions et ses perceptions. Bien que l'on reconnaisse l'aspect dysfonctionnel de la communication, les besoins et les sentiments exprimés par la personne à travers son comportement sont vus comme légitimes. On cherchera alors à l'aider plutôt qu'à la punir.

La conception féministe de la violence conjugale se situe quant à elle à l'intérieur du paradigme de l'instrumentalité. Pour Pierre Karli (1994, p. 47), « la notion de valeur instrumentale de l'agression fait allusion au fait que le répertoire comportemental [...] dote l'être vivant des moyens d'action qui lui sont nécessaires pour obtenir ce qu'il cherche et pour éviter ce à quoi il veut échapper ». Vu sous cet 
angle, le geste violent est un outil à la disposition de la personne qui l'emploie. II trouve sa source dans les attentes, les désirs et les buts plus ou moins conscients que l'individu poursuit. C'est dans cet esprit que les féministes mettent en relief le caractère intentionnel des comportements de violence conjugale (Shepard, 1991), leur effet paralysant sur les victimes et les privilèges que les agresseurs en retirent. Elles présument que ces effets sont ceux qui étaient recherchés au départ. Bien que toutes les analyses féministes attribuent une fonction instrumentale à la violence, la notion dépasse largement le cadre du féminisme. On la retrouve notamment dans les théories de l'apprentissage social qui considèrent les conséquences attendues comme déterminantes dans l'acquisition et le renforcement du comportement violent (Bandura, 1977).

La controverse autour de la valeur expressive ou instrumentale de la violence a donné lieu à des débats vigoureux, notamment entre les thérapeutes familiaux et les féministes qui travaillent avec les femmes victimes de violence (Rondeau, Brodeur et Carrier, 2001). Bien que les deux paradigmes ne soient pas facilement réconciliables, certains efforts sont faits pour tenter de trouver une position mitoyenne qui tienne compte des deux dimensions à la fois. Par exemple, Augusta-Scott et Dankwort (2002) ont récemment critiqué la conception féministe classique selon laquelle les conjoints aux comportements violents cherchent toujours à contrôler et à dominer les femmes, les motifs qu'ils invoquent pour expliquer leur comportement étant toujours considérés comme des prétextes. Les auteurs soulignent que la présomption de malhonnêteté qui sous-tend cette approche laisse peu de place pour explorer avec les hommes des scénarios de changement basés sur leur désir d'établir des relations égalitaires et satisfaisantes avec leur partenaire. Leur nouvelle approche autorise le développement de l'empathie avec les hommes en conceptualisant la violence d'une façon qui n'est ni complètement instrumentale, ni purement expressive. Cette approche se distingue du discours féministe plus traditionnel, mais aussi d'une approche comme celle que propose Dallaire (2001).

Les défenseurs des droits des hommes expliquent la présence de violence à l'intérieur des couples à partir de facteurs interactionnels, biologiques, économiques ou psychologiques. Ils rejettent toutefois les explications fondées sur une analyse des rapports sociaux de genre. Ils prennent ainsi le contre-pied de la position féministe qui définit la violence conjugale comme un problème de domination des 
hommes sur les femmes tout en excluant les autres explications possibles. Pour sortir de ce débat contradictoire, plusieurs auteurs voient maintenant la violence comme le résultat d'une interaction entre des facteurs socioculturels et des facteurs individuels qui agissent à des niveaux différents (Maiuro, Hagar, Lin et Olson, 2001). D'autres, comme O'Neil et Harway (1999), cherchent à bâtir des modèles explicatifs complexes qui intègrent les facteurs macrosociaux, les déterminants biologiques, les interactions au sein du couple et la socialisation différenciée des hommes et des femmes. Dans cette perspective multifactorielle, il y a place pour la critique des défenseurs des droits des hommes face à une vision réductionniste proposée par certaines auteures féministes. II y a cependant aussi place pour l'intégration des théories féministes à l'intérieur d'une explication globale de la violence conjugale (Lenton, 1995a; 1995b).

\section{La symétrie de la violence}

Les théories féministes soutiennent globalement: (a) que la violence conjugale est exercée principalement par les hommes contre les femmes; (b) que certaines femmes emploient la violence dans un cadre de légitime défense; et (c) que la violence des femmes contre les hommes en dehors de ce cadre défensif constitue un phénomène marginal. Les défenseurs des droits des hommes clament au contraire que la violence des femmes est parfaitement symétrique à celle des hommes. La controverse provient d'études menées auprès d'échantillons représentatifs de la population qui ont révélé des taux de victimisation semblables chez les hommes et les femmes. Dans une enquête sociale générale menée en 1999 auprès de 26000 personnes, le Centre canadien de la statistique juridique (2000) indique par exemple qu'environ 690000 Canadiennes mariées ou vivant en union de fait ont été victimes d'une forme quelconque de violence de la part de leur conjoint au cours des cinq années précédentes, alors que 549000 hommes ont vécu la même situation. Les taux quinquennaux de victimisation sont respectivement de $8 \%$ chez les femmes et de $7 \%$ chez les hommes.

Ces études soulèvent des interrogations à propos de la violence féminine et de l'universalité du modèle homme agresseur / femme victime. Elles sont toutefois insuffisantes pour conclure à la symétrie générale de la violence des hommes et des femmes, et ce, pour trois raisons : 
- Premièrement, quand on analyse les résultats de l'étude du Centre canadien de la statistique juridique (2000), on constate que les femmes rapportent des formes de violence plus graves (ex. : battues, étranglées, menacées avec une arme), qu'elles sont plus susceptibles d'être agressées à plusieurs reprises, qu'elles courent un plus grand risque de subir des blessures et qu'elles souffrent plus souvent de conséquences psychologiques négatives que les hommes.

- Deuxièmement, les études dites représentatives de la population ont leurs propres limites méthodologiques. Johnson (1995), par exemple, indique que les enquêtes nationales menées aux États-Unis ne rejoignent qu'une faible partie des femmes qui utilisent les maisons d'hébergement. Elles ne peuvent par conséquent pas être opposées aux études effectuées par les chercheures féministes auprès des femmes hébergées, puisque les deux types d'études rejoignent des populations distinctes. Straus (1999), dont les travaux inspirent souvent ceux qui avancent la thèse de la symétrie, rappelle quant à lui que les résultats des études de grande envergure sur les conflits familiaux doivent être comparés à ceux des enquêtes criminologiques démontrant que la violence conjugale est presque exclusivement un crime masculin. Alors que les premières s'intéressent aux conflits familiaux sans égard aux conséquences, les secondes accordent une grande importance à la question des blessures et des torts causés à la victime. Cette différence expliquerait le taux de victimisation globalement plus bas et la proportion beaucoup plus faible de femmes violentes que l'on retrouve dans les enquêtes criminologiques. Pour Straus, la symétrie apparente observée dans les études sur les conflits familiaux disparaît lorsque l'on considère la sévérité des effets de la violence.

- Troisièmement, Johnson et Ferraro (2000) soutiennent que les études menées auprès des échantillons représentatifs de la population produisent une illusion de symétrie, car elles ne sont pas en mesure de faire la distinction entre : (a) la violence commune, caractérisée par une fréquence faible, l'absence d'escalade et la mutualité des agressions; (b) la résistance violente où la victime réplique pour se protéger ou se venger; et (c) le terrorisme intime dans lequel l'un des conjoints exerce un contrôle général sur l'autre. Leurs propres 
recherches suggèrent que la résistance violente est très largement exercée par les femmes, alors que le terrorisme intime est essentiellement masculin. À leur avis, les recherches futures devraient mieux isoler ces différents types de violence.

Contrairement à ce que Dupuy (2000) et Dallaire (2001) affirment, les données sur l'homicide conjugal ne permettent pas non plus de démontrer la symétrie de la violence entre les deux sexes. En fait, la proportion de meurtriers et de meurtrières est d'environ de trois pour un, tant au Canada que dans la plupart des pays occidentaux où l'on tient des statistiques sur ce phénomène (Centre canadien de la statistique juridique, 2002; Wilson et Daly, 1993). II est exact de dire que les États-Unis ont fait exception à cette règle pendant plusieurs décennies, la proportion de meurtrières y étant à une époque jusqu'à deux fois supérieure à celle observée au Canada, en Australie et en Grande-Bretagne (Wilson et Daly, 1992; 1993). Cette situation a cependant changé au cours des 25 dernières années, le nombre d'hommes tués par leur conjointe au cours de cette période ayant diminué quatre fois plus rapidement que le nombre de femmes tuées par leur conjoint (Bureau of Justice Statistics, 2000). Pour Gelles (2001), cette diminution coïncide avec la mise en place des ressources d'hébergement et des politiques d'arrestation visant à protéger les femmes de la violence conjugale. L'auteur fait l'hypothèse que les Américaines commettent aujourd'hui moins d'homicides contre leur conjoint parce qu'elles disposent d'autres moyens pour échapper à la violence de ce dernier. Si cette hypothèse se révèle vraie, les mesures prises pour contrer la violence conjugale auront aussi contribué à préserver la vie des hommes. Par ailleurs, l'idée d'étudier le nombre de personnes qui meurent des suites de la violence conjugale en amalgamant homicides et suicides pose des difficultés éthiques sérieuses. Bien que le suicide des hommes et des femmes aux prises avec la violence conjugale constitue un drame social auquel il faut s'intéresser, la personne suicidaire exerce un certain contrôle sur l'issue de la situation jusqu'au moment ultime. La victime d'homicide, elle, n'a pas de choix, son destin étant totalement contrôlé par l'autre. Cette différence importante justifie un traitement séparé des deux phénomènes.

Comme on vient de le montrer, plusieurs études mettent en évidence de véritables différences dans la façon dont les individus des deux sexes font usage de violence envers leur partenaire. Cela étant dit, la 
communauté scientifique n'a pas fini de répondre à toutes les questions soulevées par les enquêtes mettant en relief la violence féminine. Dowd (2001) constate que cette question est demeurée taboue au cours des vingt dernières années et qu'elle a été très peu étudiée par comparaison avec la violence des hommes. La société lève cependant lentement les yeux sur le phénomène. Ainsi, sur le plan théorique, Straus (1999) a formulé récemment une série d'hypothèses pour expliquer la violence des femmes dans la famille. II explique que celles-ci peuvent agresser pour se défendre, pour se venger d'un conjoint, pour forcer leur partenaire à discuter des problèmes du couple ou pour contrer les attaques à leur identité. Sur le plan pratique, on a aussi vu l'apparition de services de thérapie destinés aux femmes violentes (Broué et Delisle, 2002; Delisle, 1999; Dowd, 2001). Les féministes contribuent elles aussi à la réflexion sur la violence féminine et discutent sans détours des défis que cette réalité pose à l'analyse féministe (voir par exemple Guay, 1999; Lenton, 1995b).

\section{La réponse du système judiciaire}

Dupuy (2000) présente dans son livre plusieurs histoires de cas qui témoignent des conséquences liées à une mise en accusation dans une affaire de violence conjugale. Ces conséquences sont d'autant plus graves lorsqu'il s'agit de cas documentés d'erreur judiciaire. Les exemples présentés mettent en relief la fragilité du citoyen face au pouvoir de l'État et rappellent l'importance de protéger celui-ci contre toute intervention arbitraire. Dans ce contexte, on peut voir le rôle social des défenseurs des droits des hommes comme celui d'un chien de garde défendant des principes fondamentaux dans une société démocratique. II faut cependant garder à l'esprit que ces principes ne sont pas absolus et qu'ils entrent en conflit avec d'autres valeurs sociales importantes, comme la protection des victimes.

Le système judiciaire constitue l'arène où ces principes contradictoires s'affrontent et sont soupesés. Les défenseurs des droits des hommes soutiennent toutefois que l'équilibre a été rompu, les femmes jouissant à leur avis d'un traitement préférentiel à l'intérieur du système de justice. Pour mettre cette thèse en contexte, il faut d'abord rappeler que le système judiciaire canadien traverse depuis quelques années une crise de confiance. Ainsi, dans un sondage mené auprès de 505 adultes québécois, seulement $42 \%$ des répondants ont estimé que tous sont égaux devant la loi, alors que $75 \%$ 
d'entre eux affirmaient que le système favorise les riches et que $77 \%$ croyaient que la justice dépend du juge sur qui l'on tombe (Vailles, 2000). II faut aussi rappeler que les femmes victimes de violence conjugale ont elles aussi formulé de nombreux griefs à l'égard du système judiciaire. Elles lui reprochent notamment son incapacité à faire respecter par l'accusé les ordonnances qui lui sont imposées et le manque de sévérité des sentences (Damant, Paquet, Bélanger et Dubé, 2001). L'idée que les hommes accusés bénéficient d'un traitement préférentiel a été maintes fois énoncée dans les écrits portant sur la judiciarisation de la violence conjugale (Gauthier, 2001).

Dans l'une des rares études empiriques visant à valider ou invalider l'hypothèse d'un traitement préférentiel, Gauthier (2001) a effectué une comparaison systématique du traitement réservé à des hommes accusés de violence conjugale $(n=284)$ et à des hommes inculpés pour d'autres motifs $(n=1374)$. L'auteure s'est intéressée à l'usage de la détention provisoire et à l'issue des procès dans ces dossiers soumis à la Cour du Québec de Montréal. On peut synthétiser ainsi les principaux résultats de l'étude :

- Après la comparution et l'enquête préliminaire, les hommes accusés de violence conjugale sont remis en liberté dans une plus forte proportion que ceux inculpés d'autres infractions $(82,7 \%$ comparativement à 69,5\%);

- Les facteurs qui influent sur la décision de remettre le prévenu en liberté ou non sont relativement similaires pour les deux groupes ( $p$. ex. : libérer ceux qui ont une occupation, détenir ceux qui ont des antécédents judiciaires ou une cause pendante, détenir ceux qui sont accusés de crimes graves contre la personne);

- Le tiers (33,6\%) des hommes accusés de violence conjugale ressortent des procédures avec un verdict de culpabilité, comparativement à plus des trois quarts des hommes impliqués dans les autres dossiers (82\%);

- Un plus grand nombre d'hommes accusés de violence conjugale choisissent d'aller en procès au lieu de plaider coupables, le résultat étant que les procédures se terminent plus souvent par une libération des accusations $(36,7 \%$ vs $10,8 \%)$, un acquittement $(13,7 \%$ vs $3,6 \%)$, une interruption 
des procédures $(10,2 \%$ vs $1,9 \%)$ ou un retrait des accusations $(5,9 \%$ vs $1,7 \%)$ que dans le groupe de comparaison;

- Enfin, 34,9 \% des hommes trouvés coupables de violence conjugale reçoivent une sentence carcérale, contre $71,7 \%$ des hommes reconnus coupables d'autres infractions.

Gauthier (2001) pondère le poids de ces données en indiquant que la majorité des conjoints inculpés de violence conjugale sont détenus au début de la procédure judiciaire et que plusieurs situations d'agression mineure ne seraient pas judiciarisées s'il n'y avait pas de politique de mise en accusation systématique. Considérant l'ensemble de ses observations, l'auteure souligne qu'on ne peut pas conclure à un traitement préférentiel des hommes accusés de violence conjugale. On peut toutefois ajouter qu'il n'y a pas non plus d'indication d'une persécution hitlérienne à leur égard : le système judiciaire est certes particulièrement sévère envers eux au début de la procédure judiciaire, mais la majorité s'en tire en bout de ligne sans verdict de culpabilité ou avec une sentence moins lourde que les hommes accusés d'autres crimes. L'étude de Gauthier montre l'importance de mener des études comparatives et d'effectuer des analyses nuancées du traitement judiciaire de la violence conjugale. Bien qu'elles soient intéressantes d'un point de vue phénoménologique, les recensions d'histoires de cas d'injustices ne permettent ni d'apporter les nuances appropriées ni de démontrer la discrimination systématique que les défenseurs des droits des hommes allèguent.

Selon Dupuy (2000), de nombreuses femmes font de fausses allégations de violence conjugale afin d'améliorer leur position stratégique dans les procès civils intentés contre leur conjoint. Les accusations ont ici un caractère instrumental, l'objectif étant d'éloigner le conjoint pour s'approprier les biens communs et obtenir la garde des enfants. Deux observations peuvent être faites à ce propos. D'abord, tout semble se passer comme si les objectifs visés par les femmes n'avaient rien à voir avec leur protection, les finalités de leurs actions étant définies par l'auteur à partir de l'expérience d'exclusion des hommes. Or, les études sur l'utilisation du système judiciaire par les femmes violentées montrent que les objectifs qu'elles visent sont plus diversifiés et complexes. Ces femmes portent plainte notamment pour se protéger ou faire cesser la violence, pour protéger d'autres personnes, pour briser le silence à propos de leur situation, pour que leur conjoint obtienne de l'aide, pour punir ce dernier, pour 
négocier des arrangements financiers et matériels satisfaisants lors du divorce ou de la séparation (Damant et al., 2001) et pour se réapproprier un territoire personnel dont elles ont été privées pendant leur vie avec leur conjoint violent (Wuest et Merrit-Gray, 1999). La deuxième et dernière observation porte sur la cohérence de l'analyse proposée par les défenseurs des droits des hommes. II est en effet ironique de voir Dupuy (2000) camper les allégations de violence conjugale dans une perspective instrumentale, alors que les défenseurs des droits des hommes s'emploient généralement à nier le caractère instrumental des comportements violents des hommes en contexte conjugal. La démarche féministe, qui consiste à présenter les comportements violents et le recours au système judiciaire comme autant d'instruments de pouvoir à la disposition des protagonistes, paraît à cet égard beaucoup plus cohérente.

\section{CONCLUSION}

Cet article visait d'abord à présenter une vision d'ensemble du discours des défenseurs des droits des hommes sur la violence masculine et la violence conjugale à partir de trois ouvrages québécois. L'analyse thématique met en évidence le fait que ces hommes se perçoivent comme les victimes d'un procès où ils se voient injustement accusés de violence généralisée et de misogynie. Cette image négative ternit l'image masculine et cause aux hommes des préjudices psychologiques, sociaux et matériels. Elle entraîne une répression policière et judiciaire démesurée envers les hommes accusés de violence conjugale par leur conjointe. Les auteurs étudiés attribuent ces injustices envers les hommes à la montée d'un féminisme doctrinaire et fermé à la critique. Ils entendent combattre ce nouveau sexisme en limitant la définition de la violence à ses formes les plus graves, en contestant les statistiques officielles sur la violence faite aux femmes, en décrivant la violence conjugale comme un phénomène parfaitement symétrique et en plaidant pour une plus grande tolérance à l'égard des agressions mineures. Pour mener leur combat, ils mettent sur pied des associations de défense des droits des hommes, dénoncent publiquement le discours féministe sur la violence, exercent des pressions politiques et revendiquent des budgets de recherche.

Dans la seconde partie de l'article, nous avons fait état de données scientifiques qui remettent en question les thèses des défenseurs des droits des hommes sur la symétrie générale de la violence entre 
les sexes et sur la discrimination systématique dont les hommes seraient victimes au sein du système judiciaire. Nous avons aussi tenté de montrer que les définitions courantes de la violence, la question de la violence féminine et l'équilibre entre la protection des victimes et celle des droits des accusés font depuis longtemps l'objet de débats parmi les chercheurs et les intervenants qui s'intéressent à la violence conjugale. II s'agit là de sujets complexes ou peu explorés pour lesquels il y a toujours matière à réflexion. Comme Straus (1999) l'a déjà indiqué, il est peu probable que l'on en arrive un jour à de véritables consensus autour de ces questions controversées, compte tenu des objectifs politiques différents poursuivis par les acteurs qui prennent part au débat. II faut toutefois souhaiter que la discussion se poursuive dans un esprit d'humilité et d'ouverture, en se rappelant que les théories sur les causes de la violence conjugale demeurent toujours embryonnaires, même après trente ans de recherche (Harway et O'Neil, 1999). Pour manifester cette ouverture d'esprit, les défenseurs des droits des hommes doivent à notre avis dépasser leur opposition au féminisme radical et considérer davantage l'apport théorique que le mouvement de défense des femmes violentées a fourni. 


\section{RÉFÉRENCES BIBLIOGRAPHIQUES}

AugustA-ScOTT, T. et J. DANKWORT (2002). " Partner abuse group intervention. Lessons from education and narrative therapy approaches ", Journal of Interpersonal Violence, vol. 17, $\mathrm{n}^{\circ}$ 7, p. 783-805.

BAndurA, A. (1977). Social Learning Theory, Englewood Cliffs (NJ), Prentice-Hall, $247 \mathrm{p}$.

BRouÉ, J. et L. DeLISLE (2002). " L'intervention de groupe auprès des femmes ayant des comportements violents ", dans J. Broué et C. Guèvremont (dir.), Blessures d'amour, Montréal, Option, une alternative à la violence conjugale et familiale, p. 63-68.

Bureau OF Justice Statistics (2000). Intimate Partner Violence, U.S. Department of Justice, $11 \mathrm{p}$.

CentRe CANADIEN DE LA STATISTIQUe JURIDIQUe (2000). La violence familiale au Canada: un profil statistique 2000, Statistique Canada, $\mathrm{n}^{\circ}$ 85-224-XIF au catalogue, $69 \mathrm{p}$.

CenTRE CANADIEN DE LA STATISTIQUE JURIDIQUE (2002). La violence familiale au Canada: un profil statistique 2002, Statistique Canada, $\mathrm{n}^{\circ}$ 85-224-XIF au catalogue, $56 \mathrm{p}$.

Clatterbaugh, K. (1997). Contemporary Perspectives on Masculinity. Men, Women, and Politics in Modern Society (2nd edition), Boulder (CO), Westview Press, 242 p.

Clatterbaugh, K. (2000). "Literature on the U.S. men's movements ", Journal of Women in Culture and Society, vol. 5, $n^{\circ} 3$, p. 883-894.

CÔTÉ, R. (1990). Manifeste d'un salaud, Terrebonne, Éditions du Portique, $252 \mathrm{p}$.

Dallaire, Y. (2001). Homme et fier de l'être, Québec, Éditions Option Santé, $335 \mathrm{p}$.

Damant, D., J. Paquet, J.-A. BÉlanger et M. Dubé (2001). Le processus d'empowerment des femmes victimes de violence conjugale à travers le système judiciaire, Québec, Centre de recherche interdisciplinaire sur la violence familiale et la violence faite aux femmes, 130 p., coll. «Études et analyses », $n^{\circ} 14$.

DelisLE, L. (1999). "Rompre le secret », dans J. Broué et C. Guèvremont (dir.), Intervenir auprès des conjoints violents, Montréal, Éditions Saint-Martin, p. 69-73. 
DowD, L. (2001). " Female perpetrators of partner aggression : Relevant issues and treatment », Journal of Aggression, Maltreatment and Trauma, vol. 5, $\mathrm{n}^{\circ} 2$ [ $\left.\mathrm{n}^{\circ} 10\right]$, p. $73-104$.

DULAC, G. (1991). " De la métamorphose du genre masculin », Recherches sociographiques, vol. $32, n^{\circ} 3$, p. 415-425.

DupuY, G. (2000). Coupable d'être un homme. "Violence conjugale » et délire institutionnel, Montréal, VLB éditeur, $190 \mathrm{p}$.

GAUTHIER, S. (2001). La violence conjugale devant la justice. Conditions et contraintes de l'application de la loi, Paris, L'Harmattan, 191 p., coll. «Logiques sociale ».

Gelles, R.J. (2001). Domestic Violence Offenders, Implications of Current Research for Policy and Practice. Communication présentée à la septième Conférence internationale de recherche sur la violence familiale, Portsmouth (NH), 22 au 25 juillet 2001.

Gelles, R.J. et M.A. Straus (1988). Intimate Violence. The Causes and Consequences of Abuse in the American Family, New York, Touchstone Books, Simon and Schuster Inc.

Gouvernement du QuÉBEC (1995). Politique d'intervention en matière de violence conjugale. Prévenir, dépister, contrer la violence conjugale, Québec, Gouvernement du Québec.

GuAY, F. (1999). La violence conjugale chez les lesbiennes : une recension critique, Montréal, Centre de recherche interdisciplinaire sur la violence familiale et la violence faite aux femmes, $n^{\circ} 9,86$ p., coll. "Études et analyses ».

HARWAY, M. et J.M. O'NEIL (1999). " What causes men to be violent against women? The unanswered and controversial question », M. Harway et J.M. O'Neil (dir.), What Causes Men's Violence Against Women?, Thousand Oaks (CA), Sage Publications, p. 5-11.

JoHNSON, M.P. (1995). "Patriarcal terrorism and common couple violence : Two forms of violence against women », Journal of Marriage and the Family, vol. 57 (mai), p. 283-294.

JoHNSON, M.P. et K. FERRARO (2000). « Research on domestic violence in the 1990s : Making distinctions ", Journal of Marriage and the Family, vol. 62 (novembre), p. 948-963.

KARLI, P. (1994). «L'agressivité : fatalité inhérente à notre animalité ou responsabilité majeure découlant de notre liberté? ", dans Violence et coexistence humaine: actes $d u I^{e}$ congrès mondial de l'Asevico, Montréal, Éditions Montmorency, p. 45-52. 
LENTON, R. (1995a). "Power versus feminist theories of wife abuse », Canadian Journal of Criminology, vol. 37, $\mathrm{n}^{\circ} 3$, p. 305-330.

LENTON, R. (1995b). "Feminist versus interpersonal power theories of wife abuse revisited ", Canadian Journal of Criminology, vol. $37, \mathrm{n}^{\circ} 4$, p. 567-574.

MACLEOD, L. (1987). «Pour de vraies amours : prévenir la violence conjugale », Ottawa, Conseil consultatif canadien sur la situation de la femme, $191 \mathrm{p}$.

MaIURO, R.D., T.S. HAgAR, H. LIN et N.Olso (2001). «Are current state standards for domestic violence perpetrator treatment adequately informed by research? A question of questions ", Journal of Aggression, Maltreatment and Trauma, vol. 5, $\mathrm{n}^{\circ} 2$ [no 10], p. 21-44.

MESSNER, M.A. (1998). "The limits of 'The Male Sex Role'. An analysis of the men's liberation and men's rights movements' discourse », Gender and Society, vol. 12, $\mathrm{n}^{\circ}$ 3, p. 255-276.

O'NEIL, J.M. et M. HARWAY (1999). « Preliminary multivariate model explaining the causes of men's violence against women », dans M. Harway et J.M. O'Neil (dir.), What Causes Men's Violence Against Women? Thousand Oaks (CA), Sage Publications, p. 12-17.

QualitATIVE Solution AND ReSEARCH (1997). Nud-Ist (version 4.0) [logiciel informatique], Thousand Oaks (CA), Sage Publications Software.

RONDEAU, G. (1994). "La violence familiale », dans F. Dumont, S. Langlois et Y. Martin (dir.), Traité des problèmes sociaux, Québec, Institut québécois de recherche sur la culture, p. 319-335.

RondeAu, G., N. BROdeUR et N. CARRIER (2001). L'intervention systémique et familiale en violence conjugale : fondements, modalités, efficacité et controverses, Montréal, Centre de recherche interdisciplinaire sur la violence familiale et la violence faite aux femmes, $n^{\circ} 16,94 p$., coll. «Études et analyses ».

SHEPARD, M. (1991). "Feminist practice principles for social work intervention in wife abuse », Affilia, vol. 6, $\mathrm{n}^{\circ} 2$, p. 87-93.

Straus, M.A. (1999). "The controversy over domestic violence by women », dans X.B. Arriaga et S. Oskamp (dir.), Violence in Intimate Relationships, Thousand Oaks (CA), Sage Publications, p. 17-44.

STRONG-BOAG, V. (1996). «Independent women, problematic men : Firstand second-wave anti-feminism in Canada from Goldwin Smith to Betty Steele », Histoire sociale, vol. 29, n 57, p. 1-22. 
VAILLES, F. (2000). « La justice n'est pas la même pour tous, croit une large majorité de Québécois ", Les Affaires, 26 août, p. 5.

WILSON, M. et M. DALY (1992). "Who kills whom in spouse killings? On the exceptional sex ratio of spousal homicides in the United States ", Criminology, vol. $30, \mathrm{n}^{\circ}$ 2, p. 189-215.

WILSON, M. et M. DALY (1993). "Spousal homicide risk and estrangement », Violence and Victims, vol. 8, $\mathrm{n}^{\circ}$ 1, p. 3-16.

WUEST, J. et M. MERRIT-GRAY (1999). « Not going back. Sustaining the separation in the process of leaving abusive relationships », Violence against Women, vol. 5, $\mathrm{n}^{\circ}$ 2, p. 110-133. 